\title{
Correlations Between Total Cross Sections and Slopes
}

\author{
A. F. Martini, M. J. Menon, and J. Montanha \\ Instituto de Física Gleb Wataghin, Universidade Estadual de Campinas, 13083-970 Campinas, SP, Brazil
}

Received on 15 August, 2003.

\begin{abstract}
We investigate correlations between the total cross section and the slope of the elastic differential cross section for proton-proton and antiproton-proton scattering at the highest energies. Based on the empirical behavior of these quantities as function of the energy, we select two different analytical parametrizations connecting them, and obtain the correlations through fits to the experimental data available. We present and discuss practical uses of extrapolations and interpolations of the results. In the former case we refer to the estimation of the proton-proton total cross sections from the proton-air cross sections (obtained from cosmic-ray experiments), and in the later case, we critically discuss the recent measurement of the slope parameter at the BNL RHIC at $200 \mathrm{GeV}$ by the pp2pp Collaboration.
\end{abstract}

\section{Introduction}

For particle-particle and antiparticle-particle interactions at high energies (c.m. energy $\sqrt{s}$ above $10 \mathrm{GeV}$ ), protonproton $(p p)$ and antiproton-proton $(\bar{p} p)$ are the processes corresponding to the highest energy values with available data. In accelerator experiments $p p$ scattering has been investigated in detail up to $\sqrt{s} \sim 63 \mathrm{GeV}$ (CERN ISR) and $\bar{p} p$ up to $\sqrt{s} \sim 2 \mathrm{TeV}$ (Fermilab Tevatron). Currently, at BNL RHIC, $p p$ collisions at $\sqrt{s}=200 \mathrm{GeV}$ are taking place, and the first measurement of the elastic slope parameter has already been reported [1]. However, the differences between the highest energies reached, namely $(\sqrt{s})_{\max }^{\bar{p} p} \sim 30(\sqrt{s})_{\max }^{p p}$ in the past and, presently, $(\sqrt{s})_{\max }^{\bar{p} p} \sim 9(\sqrt{s})_{\text {max }}^{p p}$, rend difficult a comparative investigation of both reactions.

On the other hand, experimental information on the proton-proton total cross section exist from cosmic-ray experiments, in the interval $\sqrt{s}=6-40 \mathrm{TeV}$. However, the procedure to obtain this quantity is strongly model dependent and different models lead to different and discrepant results [2]. The steps from the experimental determination of the shower attenuation length (through analysis of extensive air showers) up to the extraction of the $p p$ total cross section are discussed in detail in Ref. [3]. For our purposes, we only recall that, in the last step, the $p p$ total cross section is obtained from the $p$ - air total cross section by means of the Glauber multiple diffraction formalism, which connects the corresponding scattering amplitudes. The $p p$ amplitude is parametrized by

$$
F_{e l}^{p p}(s, t) \propto \sigma_{t o t}^{p p}(s) \exp \left\{\frac{B(s) t}{2}\right\},
$$

where $B(s)$ is the slope of the forward differential cross section,

$$
B(s)=\frac{d}{d t}\left[\ln \left(\frac{d \sigma_{e l}^{p p}}{d t}\right)\right]_{t=0}
$$

and from the Optical Theorem the total cross section is expressed by

$$
\sigma_{t o t}^{p p}(s)=\frac{I m F_{e l}^{p p}(s, t=0)}{s} .
$$

One of the sources of the model dependence is the connection between $\sigma_{t o t}^{p p}$ and $B$, in Eq. (1) and this is the point we are mainly interested in. For example, as discussed in detail in Ref. [2], the Fly's Eye Collaboration using the geometrical scaling model $\left(\sigma_{\text {tot }}^{p p} \propto B\right)$, obtained $\sigma_{\text {tot }}^{p p}=$ $120 \pm 15 \mathrm{mb}$ at $\sqrt{s}=30 \mathrm{TeV}$; on the other hand, Gaisser, Sukhatme and Yodh, making use of the Chou and Yang prescription connecting $\sigma_{\text {tot }}^{p p}$ and $B$, estimated $\sigma_{\text {tot }}^{p p} \sim 175 \pm$ $34 \mathrm{mb}$ at $\sqrt{s}=40 \mathrm{TeV}$. Obviously, these two results are in complete disagreement [2].

In this communication we present and discuss correlations between $\sigma_{\text {tot }}^{p p}$ and $B$ whose extrapolations may allow a better determination of the $\sigma_{\text {tot }}^{p p}$ from $\sigma_{t o t}^{p-a i r}$. We also make use of interpolation of these parametrizations in order to critically investigate the recent measurement of the slope parameter by the pp2pp Collaboration.

The manuscript is organized as follows. In Sec. II we discuss the experimental data to be analyzed and also the strategies used in the selection of the parametrizations. In Sec. III we present the results of the fits, together with extrapolations to cosmic-ray energies. In Sec. IV we critically discuss the recent measurement of the slope parameter at $\sqrt{s}=200 \mathrm{GeV}$, showing the discrepancies with the parametrizations and experimental data available. The conclusions and final remarks are the contents of sec. V.

\section{Experimental data and parametriza- tions}

Fig. 1 shows the experimental data on $\sigma_{t o t}(s)$ and $B(s)$ at the same energy, for $p p$ and $\bar{p} p$ elastic scattering, from ac- 
celerator experiments and above $\sqrt{s}=10 \mathrm{GeV}[4,5]$. We have selected the data above the region of Coulomb-nuclear interference and below the "break" in the hadronic slope at the diffraction peak (localized at $|t| \sim 0.2 \mathrm{GeV}^{2}$ at the ISR and Collider energies), namely $0.01<|t|<0.20 \mathrm{GeV}^{2}$. In this region, the differential cross section data are well fitted by a single exponential and therefore there is no change in the slope associated with the $t$-dependence, but only with the energy [5].
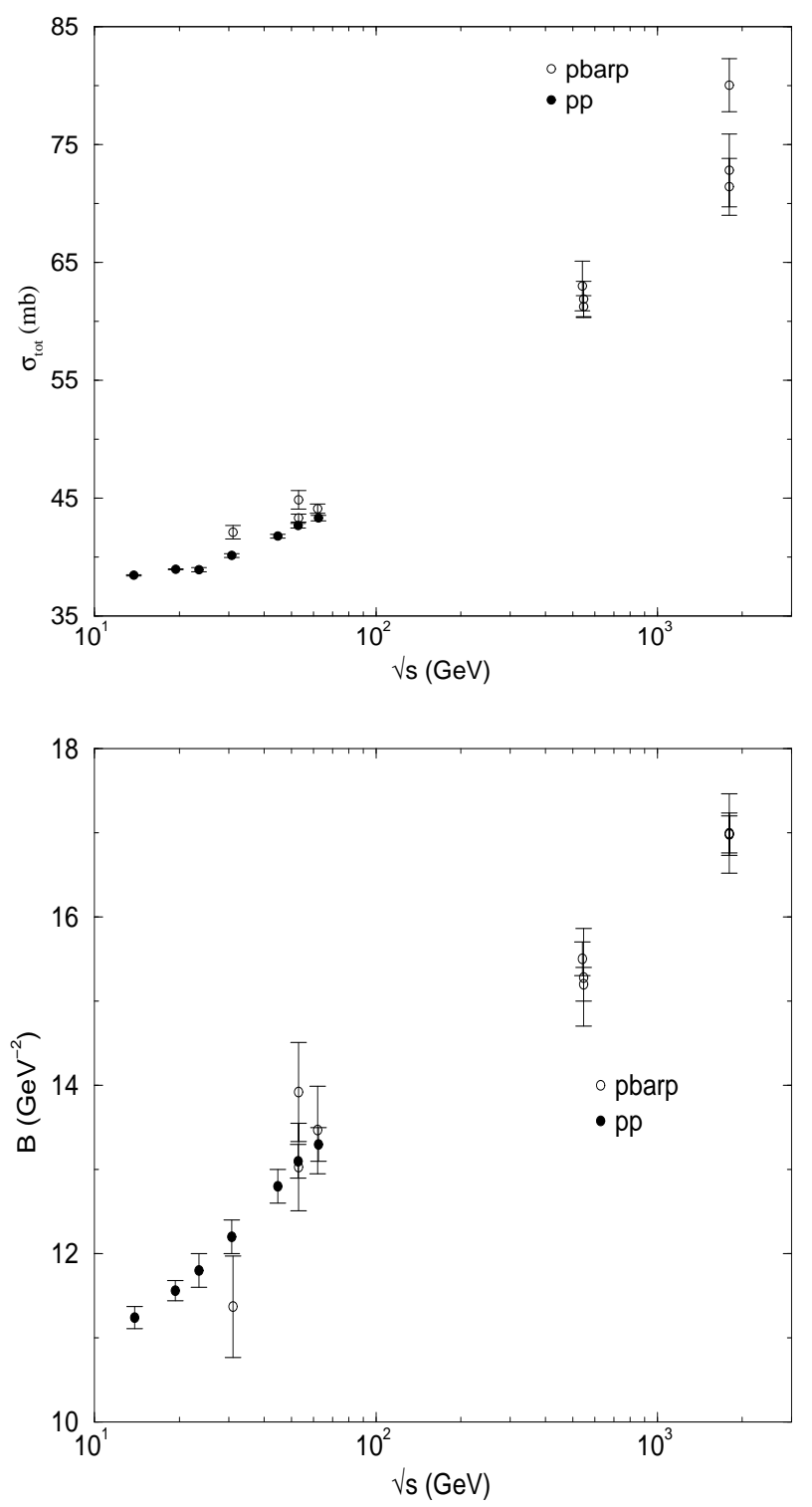

Figure 1. Experimental data on total cross section and the corresponding values of the slope at each energy, for $p p$ and $\bar{p} p$ elastic scattering above $10 \mathrm{GeV}$ [4].

Our choices for the parametrizations are based on the following observations. From Fig. 1, it is empirically evident that the slope parameter increases linearly with the logarithm of the c.m. energy and the total cross section follows some second degree polynomial in $\ln s$. Besides agreement with the above results, suitable analytical parametrizations, connecting $\sigma_{\text {tot }}(s)$ and $B(s)$, should also be efficient in the individual description of $\sigma_{t o t}$ as function of the energy. For these reasons, we consider two analytical forms, associated with two different parametrizations, one introduced by Donnachie and Landshoff (DL) [6], $\sigma_{t o t}^{p p}(s)=X s^{\epsilon}+Y s^{-\eta}$, $\sigma_{\text {tot }}^{\bar{p} p}(s)=X s^{\epsilon}+Z s^{-\eta}$ and the other by Kang and Nicolescu (KN) [7] : $\sigma_{\text {tot }}^{p p}(s)=A_{1}+B_{1} \ln s+k \ln ^{2} s, \sigma_{\text {tot }}^{\bar{p} p}(s)=$ $A_{2}+B_{2} \ln s+k \ln ^{2} s+2 R s^{-1 / 2}$. The differences between these parametrizations are well known and have been recently reviewed in [2].

Based on the above observations, we consider analytical forms connecting $\sigma_{t o t}(s)$ and $B(s)$ that follow the DL and $\mathrm{KN}$ parametrizations, that is, we identify $B(s)$ with $\ln s$ in these equations. Several tests are possible, with different constraints for the free fit parameters. In this communication we shall consider the results with the reasonable assumption that, asymptotically, $p p$ and $\bar{p} p$ scattering follow the same dependence on the energy. However, the differences in the structure of the parametrizations allow different asymptotic behaviors, as we shall show. Specifically, we introduce the following forms and notation connecting $\sigma_{t o t}(s)$ and $B(s)$ :

- Parametrization $a$

$$
\begin{aligned}
\sigma_{t o t}^{p p} & =a_{1} e^{a_{2} B}+a_{3} e^{-a_{4} B} \\
\sigma_{t o t}^{\bar{p} p} & =a_{1} e^{a_{2} B}+a_{5} e^{-a_{6} B}
\end{aligned}
$$

- Parametrization $b$

$$
\begin{aligned}
& \sigma_{\text {tot }}^{p p}(s)=b_{1}+b_{2} B+b_{3} B^{2} \\
& \sigma_{\text {tot }}^{\bar{p} p}(s)=b_{1}+b_{2} B+b_{3} B^{2}+b_{4} e^{-B / 2}
\end{aligned}
$$

Obviously, these are strictly mathematical choices, having nothing to do with the physics behind the DL and KN parametrizations.

\section{Fitting results and extrapolations to cosmic-ray energies}

Fits to the experimental data, displayed in Fig. 1, through Eqs. (2) and (3) have been performed with the CERNMinuit program. Table I shows the results of simultaneous fits to $p p$ and $\bar{p} p$ data, and Fig. 2 shows the parametrizations $a$ and $b$ together with the experimental data.

Due to the large error bars (mainly in the $B$ parameter) and the small number of points, the $\chi^{2} / \mathrm{DOF}$ is a bit high. However, Fig. 2 shows that the data are well described in both cases.

In Fig. 3 we plot the extrapolations of both parametrizations up to values of the total cross sections typical of cosmic-ray experiments, $\sigma_{\text {tot }} \sim 200 \mathrm{mb}$ (Sec. I). We see that above $\sigma_{t o t}=70-80 \mathrm{mb}$ (typical of Tevatron energies), the parametrizations diverge fast. Since the DL parametrization (a) eventually violates the Froissart-Martin bound, we understand that the most truthfull and also conservative result for extrapolations concerns that associated with the $\mathrm{KN}$ parametrization $(b)$. 
TABLE I. Fit results

\begin{tabular}{lclc}
\hline \hline \multicolumn{2}{c}{ parametrization a } & \multicolumn{2}{c}{ parametrization b } \\
\hline$a_{1}(\mathrm{mb})$ & $0.674 \pm 0.014$ & $b_{1}(\mathrm{mb})$ & $163.5 \pm 0.3$ \\
$a_{2}\left(\mathrm{GeV}^{2}\right)$ & $0.272 \pm 0.001$ & $b_{2}\left(\mathrm{mbGeV}^{2}\right)$ & $-22.65 \pm 0.03$ \\
$a_{3}(\mathrm{mb})$ & $101 \pm 1$ & $b_{3}(\mathrm{mbGeV})$ & $1.027 \pm 0.002$ \\
$a_{4}\left(\mathrm{GeV}^{2}\right)$ & $0.128 \pm 0.001$ & $b_{4}(\mathrm{mb})$ & $631 \pm 127$ \\
$a_{5}(\mathrm{mb})$ & $179 \pm 17$ & - & - \\
$a_{6}\left(\mathrm{GeV}^{2}\right)$ & $0.168 \pm 0.008$ & - & - \\
No. DOF & 11 & No. DOF & 13 \\
$\chi^{2} / \mathrm{DOF}$ & 7.9 & $\chi^{2} / \mathrm{DOF}$ & 8.0 \\
\hline \hline
\end{tabular}
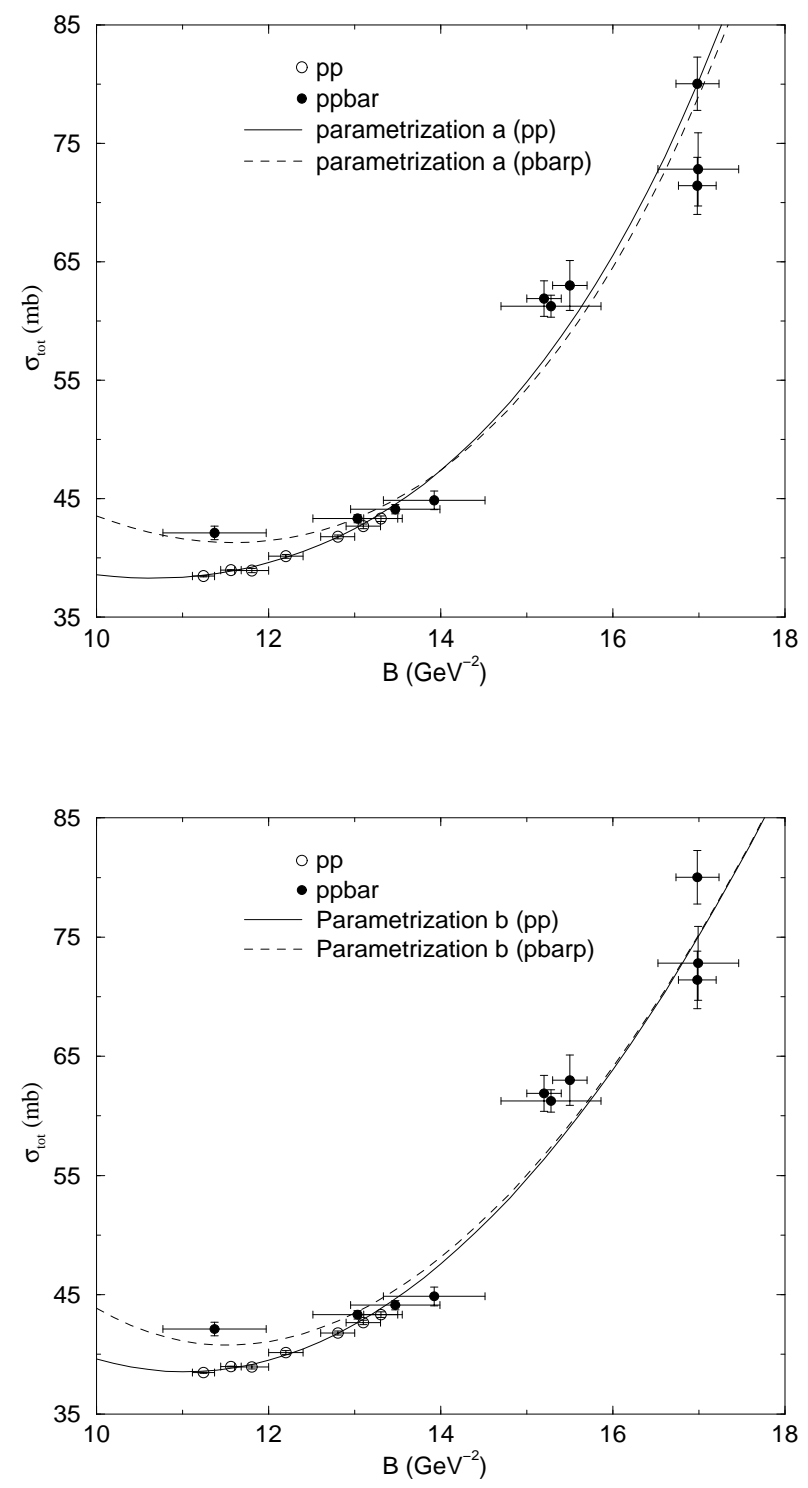

Figure 2. The total cross section in terms of the slope and the fitted parametrizations $a$ (above) and $b$ (below).

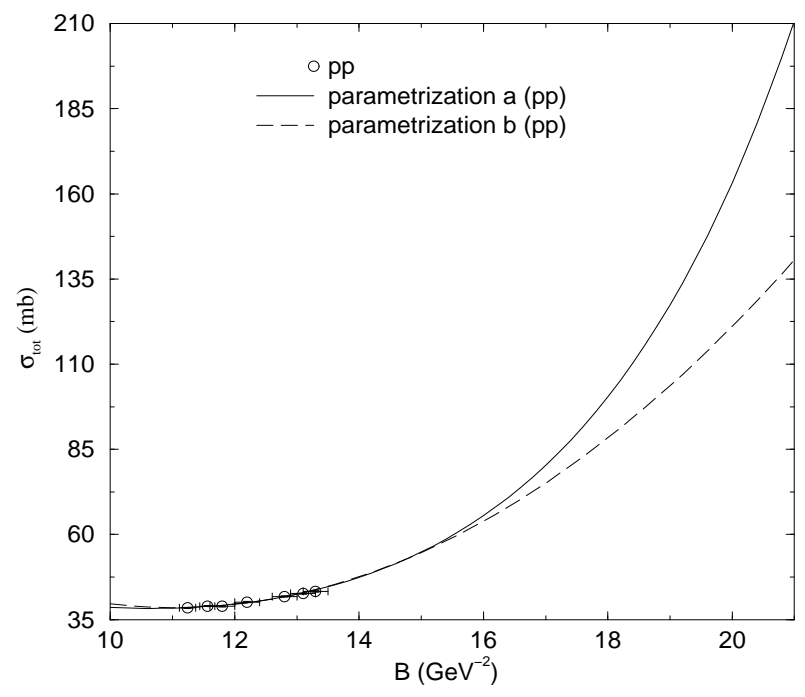

Figure 3. Extrapolation of the parametrizations up to values of the total cross section typical of cosmic-ray energies.

\section{The slope parameter at $200 \mathrm{GeV}$}

In this section we discuss the recent measurement of the slope parameter by the pp2pp Collaboration at $\sqrt{s}=200$ $\mathrm{GeV}$ [1]. The $B$ value has been extracted from fit to the differential cross section data in the $t$ range $0.010 \leq|t| \leq 0.019$ $\mathrm{GeV}^{2}$. The corresponding amplitude has contributions from the Coulomb amplitude, nuclear amplitude and the interference between them. It is parametrized by [1]

$$
\begin{aligned}
\frac{d \sigma}{d t} & =4 \pi(\hbar c)^{2}\left(\frac{\alpha G_{E}^{2}}{t}\right)^{2}+\frac{1+\rho^{2}}{16 \pi(\hbar c)^{2}} \sigma_{t o t}^{2} e^{-B|t|} \\
& -(\rho+\Delta \Phi) \frac{\alpha G_{E}^{2}}{|t|} \sigma_{t o t} e^{-\frac{1}{2} B|t|}
\end{aligned}
$$

The fit parameters are the slope $B$ and a normalization constant. The input values for $\sigma_{\text {tot }}$ and $\rho$ used by the authors were $51.6 \mathrm{mb}$ (obtained from the DonnachieLandshoff model) and 0.13 (fit by the UA4/2 Collaboration), respectively. The resulting slope parameter was $B=16.3$ \pm 1.6 (stat.) \pm 0.9 (syst.) $\mathrm{GeV}^{-2}$. Adding in quadratures the error reads $\pm 1.8 \mathrm{GeV}^{-2}$. 
According to the above result, at $\sqrt{s}=200 \mathrm{GeV}$, the value of the total cross section corresponding to $B=16.3$ $\pm 1.8 \mathrm{GeV}^{-2}$ is $\sigma_{t o t}=51.6 \mathrm{mb}$. On the other hand, if we use this $B$ value as input in our parametrizations, we infer: $\sigma_{\text {tot }}^{p p}(200 \mathrm{GeV})=69_{-19}^{+33} \mathrm{mb}$ from parametrization $a$ and $\sigma_{\text {tot }}^{p p}(200 \mathrm{GeV})=67_{-16}^{+23} \mathrm{mb}$ from parametrization $b$.

These three "peers" of points are plotted in Fig. 4, together with the experimental data. The above values of $\sigma_{\text {tot }}^{p p}(200 \mathrm{GeV})$ from our parametrizations are displayed in Fig 5, together with all the experimental information presently available on total cross sections, above $10 \mathrm{GeV}$ (see [2] for a review and numerical values concerning the cosmic-ray estimates).

From Fig. 2 and 4 we see that the point from the pp2pp Collaboration is in disagreement with our parametrizations and also with the general behavior of the experimental data. If this value of the slope is correct, then new physics is suggested: the $p p$ correlation between $\sigma_{t o t}$ and $B$ follows a linear dependence and the $\bar{p} p$ correlation a quadratic dependence, with a crossing nearly $B \sim 14 \mathrm{GeV}^{-2}$ and $\sigma_{\text {tot }} \sim$ $45 \mathrm{mb}$. These Figures also show that if $B(200 \mathrm{GeV})$ is used as input, our parametrization predicts cross sections that are in agreement with the general trend of the experimental data on $\sigma_{t o t}$ and $B$, suggesting, in this case, no need for new physics. However, as shown in Fig. 5, these inferred values for the total cross sections are much higher than generally expected for $p p$ scattering. Moreover, looking at the estimations from cosmic-ray experiments, these values are in qualitative agreement with the fastest increasing scenario for $\sigma_{t o t}(s)$, namely, the results by Gaisser-Sukhatme-Yodh (GSY) and Nikolaev [2]. In this case, the experimental information indicate a crossing, with $\sigma_{\text {tot }}^{p p}$ becoming greather than $\sigma_{\text {tot }}^{\bar{p} p}$ above $\sqrt{s} \sim 70-80 \mathrm{GeV}$, and, once more, new physics is suggested.

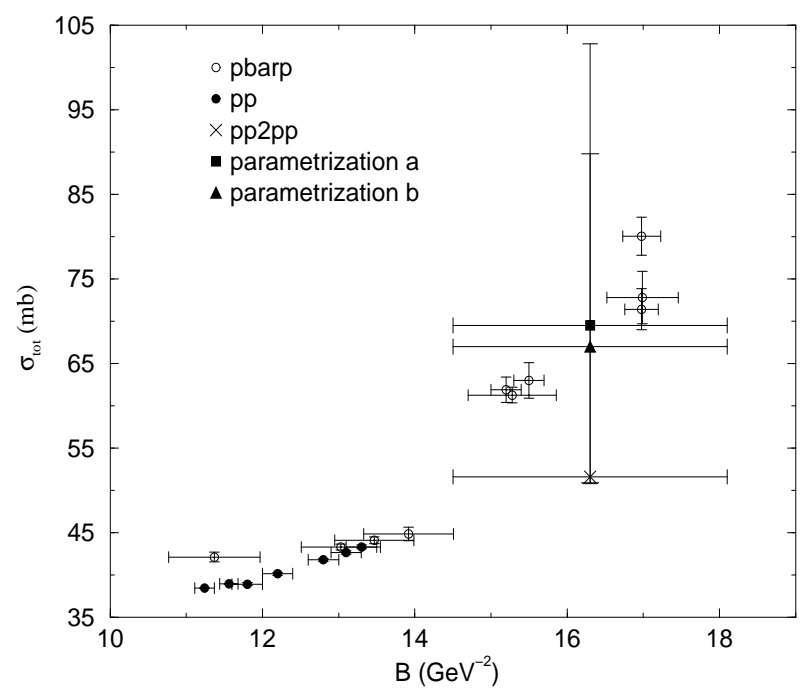

Figure 4. Experimental data on total cross section and the corresponding slope together with the input cross section value and the extracted slope by the pp2pp Collaboration.

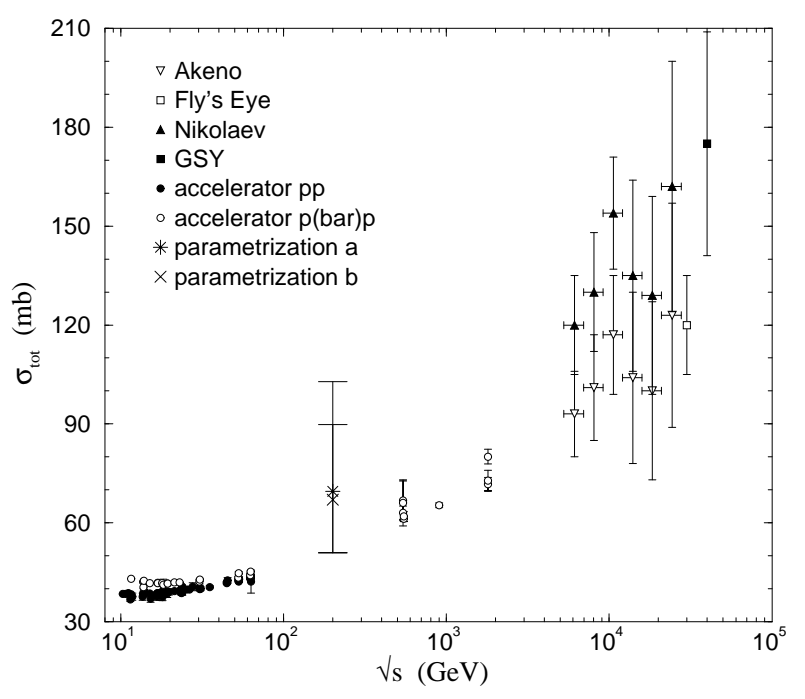

Figure 5. The total cross section and the limits for $\sigma_{p p}^{t o t}$ at $\sqrt{s}=$ $200 \mathrm{GeV}$ using parametrizations a and b, Eqs. (2) and (3) (see Ref. [2] for detailed information on the estimations at cosmic-ray energies).

\section{Conclusions}

In this communication we made use of two different parametrizations connecting the experimental data on the total cross section and the slope parameter. Extrapolations to cosmic-ray energies with parametrization $b(\mathrm{KN})$ may be useful in the determination of the $p p$ total cross section from $p$-air cross section, allowing to connect $\sigma_{t o t}-B$ in an almost model independent way. We are presently investigating this subject.

We showed that the combination $B=16.3 \pm 1.8 \mathrm{GeV}^{-2}$ and $\sigma_{t o t}=51.6 \mathrm{mb}$, indicated by the pp2pp analysis, is in disagreement with the general trend for the behaviors of $\sigma_{t o t}$ and $B$. If this "peer" is correct, new physics is necessary. Using the above $B$ value as input in our parametrizations, the corresponding values of the total cross sections show agreement with the $\sigma_{t o t}$ versus $B$ data. However, these inferred values for $\sigma_{t o t}$ indicate new physics when plotted as function of the energy.

It should be noted that the slope parameter at $200 \mathrm{GeV}$ has been determined in a relatively small region of the momentum transfer $\left(0.010 \leq|t| \leq 0.019 \mathrm{GeV}^{2}\right)$, just above the region of Coulomb-nuclear interference, and may be that its value has been overestimated. However, if the $B(200 \mathrm{GeV})$ measurement is correct and represents an hadronic quantity, its high value may indicate a "break" in the slope near 0.02 $\mathrm{GeV}^{2}$, a phenomenon that was never observed in both $p p$ and $\bar{p} p$ scattering, at $\sqrt{s} \leq 62.5 \mathrm{GeV}$ and $\sqrt{s} \leq 1.8 \mathrm{TeV}$, respectively. Once more, we conclude that new physics is necessary.

\section{Acknowledgments}

We are thankful to FAPESP for financial support (Contract N. 01/08376-2 and 00/04422-7). 


\section{References}

[1] S. Bültmann et al. (pp2pp Collaboration), arXiv:nuclex/0305012 v4.

[2] R. F. Avila, E. G. S. Luna, and M. J. Menon, Phys. Rev. D 67, 054020 (2003).

[3] T. K. Gaisser, U. P. Sukhatme, and G. B. Yodh, Phys. Rev. D 36, 1350 (1987); R. Engel, T.K. Gaisser, P. Lipari, and T. Stanev, Phys. Rev. D 58, 014019 (1998).

[4] A. S. Carrol et al., Phys. Lett. B61, 303 (1976); A. S. Carrol et al., Phys. Lett. B80, 423 (1979); U. Amaldi, and K. R. Schubert, Nucl. Phys. B166, 301 (1980); V. Bartenev et al., Phys. Rev. Lett. 31, 1089 (1973); N. A. Amos et al., Nucl. Phys. B262, 689 (1985); A. Breakstone et al., Nucl. Phys. B248,
253 (1984); M. Ambrosio et al., Phys. Lett. B115, 495 (1982); C. Augier et al., Phys. Lett. B344, 451 (1995); C. Augier et al., Phys. Lett. B316, 448 (1993); M. Bozzo et al., Phys. Lett. B147, 392 (1984); F. Abe et al., Phys. Rev. D50, 5518 (1994); F. Abe et al., Phys. Rev. D50, 5550 (1994); N. A. Amos et al., Phys. Rev. Lett. 68, 2433 (1992);C. Avila et al., Phys. Lett. B537, 41 (2002).

[5] R. Castaldi and G. Sanguinetti, Ann. Rev. Nucl. Part. Sci. 35, 351 (1985)

[6] A. Donnachie and P. V. Landshoff, Z. Phys. C 2, 55 (1979); Phys. Lett. B 387, 637 (1996).

[7] K. Kang and B. Nicolescu, Phys. Rev. D 11, 2461 (1975). 